\title{
Migração interna e pressão arterial no Rio Grande do Sul *
}

\author{
Maria do Carmo Leal ${ }^{* *}$ \\ Eduardo de Azeredo Costa * \\ Carlos Henrique Klein ** \\ Célia Landmann Szwarcwald ** \\ Paulo Cesar Rosito Barata **
}

A cross-sectional survey was carried-out in 1978 in Rio Grande do Sul, the southermost state of Brazil (population: about 8,000,000 inhabitants in 1978).

The state was divided into four strata, each one being a district geo-economic area: Porto Alegre, the state capital; Metropolitan Belt, including 13 counties around Porto Alegre; Urban Interior, the non-metropolitan urban areas of the state; Rural Interior, composed of all non-urban areas outside the Metropolitan Belt.

In each stratum a probability sample was selected in multiple stages. A total of 2.056 households were successfully contacted $194.7 \%$ of the 2.172 households selected).

This article presents an analysis of migration between the four strata and its relation with blood pressure.

The lowest blood pressure level was found in the Rural Interior, and the highest in the Metropolitan Belt.

The mean diastolic blood pressure was about the same in each stratum for migrants and non-migrants.

Both residents in Porto Alegre and Metropolitan Belt born in the Rural Interior were found to have higher mean blood pressure than non-migrants of the Rural Interior. On the other hand, persons who migrated within the rural stratum (from one rural area to another) were found to have a low blood pressure level, not differing from the non-migrants.

Migrants from the Rural Interior, residing in urban areas were found to have blood pressure levels that increased with the period of time since migration.

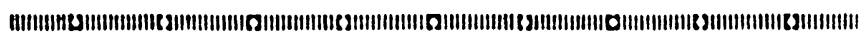

* Este trabalho é um

desdobramento da pesquisa "Características Epidemiologicas da Hipertensão Arterial no Rio Grande do Sul", financiada pelo Convênio FIOCRUZ/FINEP/ SECRETARIA DE SAÜDE DO RIO GRANDE DO SUL, realizada pelo Departamento de Epidemiologia e Métodos Quantitativos em Saúde. Escola Nacional de Saúde Pública, Fundação Oswaldo Cruz.

** Escola Nacional de Saúde Pública - FIOCRUZ - RJ

\section{INTRODUÇÃO}

$\mathrm{O}$ estudo da hipertensão arterial muito se tem enriquecido com a incorporação das variáveis sociais na discussão da determinação da doença.

$\mathrm{O}$ reflexo dos movimentos migratórios sobre a saúde, particularmente no que se refere às doenças crônico-dege- 
nerativas como a hipertensão arterial, tem sido tema de muitos debates.

No en tanto, os estudos realizados em populações migrantes apresentam dificuldades metodológicas. $O$ acompanhamento de indivíduos desde uma fase anterior à migração é difícil, pois os locais de destino dos migrantes costumam ser variados. Por outro lado, nos estudos onde os migrantes são encontrados residindo fora do local de nascimento, desconhecem-se as características destes na fase anterior à migração. Por isto na análise das relações entre migração e doença, questões polêmicas como seletividade e predisposição encontram campo fértil ${ }^{5}, 15,24$.

Muitos estudos sobre hipertensão arterial foram realizados em populações migrantes. Resultados relativamente uniformes são relatados pelos autores: quando ocorre migração de indivíduos que habitam comunidades mais simples para sociedades complexas, modemas, os niveis pressóricos encontrados nestes migrantes são mais elevados que os dos seus pares do local de nascimento ${ }^{6,14,17}$. Mudanças de padrões culturais e de hábitos alimentares são apresentadas como possíveis fatores explicativos deste comportamento ${ }^{2}, 7,10$.

A migração possibilita mudanças sócio-culturais e, portanto, o encontro com estímulos ambientais novos, determinando a ocorrência de reações de estresse freqüentes.

Reações de estresse freqüentes podem contribuir para o desenvolvimento de doenças adaptativas ${ }^{19}, 20$, dentre elas a hipertensão arterial. Assim sendo, os migrantes estariam particularmente predispostos à hipertensão arterial.

Scoth ${ }^{18}$ discute criteriosamente o significado do estímulo estressor e questiona a sua universalidade. Assim, ele pergunta se um estímulo pode ser estressante independentemente das formas de organização social de uma comunidade.

Da mesma forma, outros autores, dentre eles Cassel ${ }^{1}$, questionam a migração em si como causadora de estresse. Pode-se portanto questionar também a migração como fator de risco para a hipertensão arterial.

A maioria dos trabalhos que se dedicam a estudar as relações entre migração e pressão arterial o faz em comunidades isoladas geográfica e culturalmente. Em sociedades complexas, com as relações sociais muito desenvolvidas como a nossa, estes estudos são poucos. E, quando existem, abordam predominantemente os deslocamentos rurais-urbanos.

Neste trabalho, se analisa a migração entre cidades, de cidades para a área rural, de uma área rural para outra e da área rural para diferentes tipos de cidades. Importa saber que as áreas rurais nas sociedades atuais mantêm um contato intenso com as cidades e se tornam economicamente de- 
pendentes dela. Disto resultou uma maior semelhança entre os hábitos, dentre eles o consumo de alimentos.

\section{METODOLOGIA}

Este estudo seccional foi realizado no Rio Grande do Sul, no ano de 1978.

Utilizou-se uma amostra estratificada, onde o Estado do Rio Grande do Sul foi dividido em quatro estratos, cada um dos quais representando uma área geoeconômica distinta:

PORTO ALEGRE, a capital do Estado, a área mais urbanizada onde o setor terciário da economia está concentrado. Foi um grande pólo receptor de migrantes do Estado, tendo o fluxo diminuído nas três últimas décadas ${ }^{8},{ }^{9}$.

CINTURÃO METROPOLITANO, formado por 13 municípios que circundam Porto Alegre, onde há o predomínio do setor secundário da economia. De 1960 para cá, os Censos Demográficos vêm mostrando que esta área teve um crescimento populacional grande, às custas de uma intensa migração interna.

INTERIOR URBANO, composto pela área urbana nãometropolitana do Estado. Esta área apresenta grande variação quanto aos movimentos migratórios, desde cidades em franco processo de crescimento demográfico até aquelas com crescimento negativo por emigração.

INTERIOR RURAL, composto por todas as áreas não definidas como metropolitanas ou urbanas. Aí está localizado o setor primário da economia, e nesta área tem se processado uma emigração de forma persistente e acentuada nos últimos 40 anos.

Em cada estrato, foi selecionada uma amostra probabilística com um esquema de sorteio em estágios múltiplos. Para uma descrição mais detalhada do desenho e procedimento amostral, ver Costa ${ }^{4}$ e Klein $^{12}$.

Um total de 2.056 domicílios foram entrevistados (94,7\% dos 2.172 domicílios selecionados) e foram estudados $94,4 \%$ dos 4.835 habitantes da faixa etária entre 20 e 74 anos. Assim sendo, a amostra final consisțiu em 4.565 indivíduos desta faixa etária. Cada estrato correspondeu a aproximadamente $1 / 4$ da amostra total.

Para cada pessoa incluída na amostra, foram tomadas as medidas de pressão arterial, pulso radial, peso, altura, prega cutânea e circunferência do braço.

Uma atenção especial foi dada à medida da pressão arterial. Os examinadores foram treinados pelo método do Prof. Geoffrey Rose ${ }^{16}$. Os instrumentos utilizados foram esfigmomanômetro Tycos de coluna de mercúrio (HRI 8104509701), equipado com manuguito padrão, e estetoscópio da mesma marca (HRI 2104-509701), modelo Howell. O esfigmomanômetro foi modificado com o objetivo de reduzir 
ao máximo os erros de tendenciosidade cometidos na leitura da pressão arterial ${ }^{4}, 12,16$.

O questionário aplicado a cada pessoa incluía questões sobre a história da migração.

Foi considerado migrante o indivíduo que estava residindo por mais de seis meses fora do local do nascimento ou aquele que se encontrava no local de nascimento, mas passou fora dele um período superior a seis meses.

O não-migrante ou natural seria, portanto, o indivíduo que nunca residiu por mais de seis meses fora do local de nascimento.

Este conceito de não-migrante é mais restritivo que o utilizado habitualmente. Entretanto, para discutir a associação entre migração e pressão arterial, importa que os naturais se constituam em um grupo realmente distinto do ponto de vista do fenômeno que se deseja estudar, ou seja, a migração. Por isso, foi definido como natural o indivíduo que nunca experimentou migrar. Se, por um lado, esta definição tornou mais puro o grupo dos não-migrantes, ela heterogeneizou ainda mais o grupo dos migrantes. $\mathrm{Na}$ tentativa de minimizar este problema, os migrantes foram agrupados por local de nascimento com os mesmos critérios que foram utilizados para definição dos estratos amostrais. Assim sendo, foi possível recuperar os grupos que nasceram em um determinado estrato amostral, saíram e retornaram ao mesmo estrato, sendo esta migração denominada de intra-estrato.

Dessa forma, foram definidos os seguintes grupos de migração:

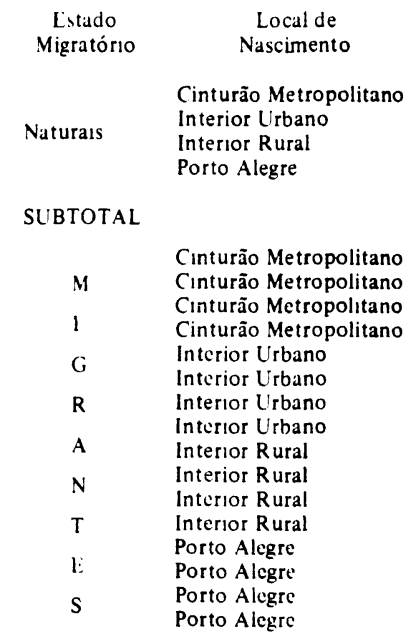

\begin{tabular}{lr}
\multicolumn{1}{c}{$\begin{array}{c}\text { Recal de } \\
\text { Residência }\end{array}$} & $\begin{array}{r}\text { Número de } \\
\text { Indivíduos }\end{array}$ \\
Cinturão Metropolitano & 143 \\
Interior Urbano & 322 \\
Interior Rural & 501 \\
Porto Alegre & 309 \\
& 1.275 \\
Cinturão Metropolitano & 70 \\
Interior Urbano & 3 \\
Interior Rural & 1 \\
Porto Alegre & 29 \\
Cinturão Metropolitano & 185 \\
Interior Urbano & 207 \\
Interior Rural & 23 \\
Porto Alcgre & 343 \\
Cinturão Metropolitano & 582 \\
Interior Urbano & 591 \\
Interior Rural & 527 \\
Porto Alegre & 300 \\
Cinturão Metropolitano & 86 \\
Interior Urbano & 11 \\
Interior Rural & 4 \\
Porto Alegre & 46 \\
& \\
&
\end{tabular}

$\mathrm{O}$ número de indivíduos nascidos no Cinturão Metropolıtano e Porto Alegre que migraram para o Interior Urbano e Interior Rural foi muito pequeno: devido a isto, eles foram suprımidos da análise. 
Foi escolhida como medida de pressão arterial a 2 a leitura feita nos individuos sentados, aproximadamente 30 minutos após a chegada ao domicílio. Optou-se por trabalhar neste estudo com a média de pressão arterial diastólica, fase $\mathrm{V}$ dos sons de Korotkow, correspondente ao desaparecimento dos sons arteriais.

A relação entre obesidade e hipertensão arterial, bem como entre aumento de peso e aumento dos níveis pressóricos tem sido descrita em vários trabalhos ${ }^{3}, 11$. Um índice de massa corpórea ou índice de Quetelet [(Peso/Altura $\left.\left.^{2}\right) \times 1000\right]^{22}$ foi utilizado com o objetivo de permitir a padronização e posterior comparabilidade de pressão arterial entre os grupos migrantes.

Para analisar diferenças de pressões médias em cada esta. do migratório, utilizou-se uma análise de covariâncias, considerando-se como variável-critério a pressão diastólica ( 2 a medida), como covariáveis idade e Indice de Quetelet e como fatores o sexo e o estado migratório com os respectivos níveis citados no quadro acima.

A pressão arterial diastólica $Y_{\mathrm{ijh}}$ foi descrita através do modelo linear:

$Y_{i j h}=\delta_{h}+\alpha_{h}(\text { idade })_{i j h}+\gamma_{h}$ (quetelet $)_{i j h}+\beta_{j h}+e_{i j h}$ onde $i, j$, e h representam, respectivamente, indivíduo, grupo de migração e sexo.

$\mathrm{O}$ efeito de cada estado migratório j sobre a média da pressão arterial diastólica em cada sexo $\mathrm{h}$ foi medido pelos parâmetros $(h=1,2 ; j=1,2 \ldots 16)$. Para estimativa de contrastes com os parâmetros do modelo linear e seus desviospadrão, utilizou-se o programa Multivariate General Linear Model (MGLM) ${ }^{23}$.

\section{RESULTADOS}

No Gráfico 1, podem ser vistas as médias brutas de pressão arterial diastólica por local de residência, para ambos os sexos. Verifica-se que os valores se encontram na seguinte ordem decrescente: Cinturão Metropolitano, Porto Alegre, Interior Urbano e Interior Rural.

Tendo em vista que estes dados não estão discriminados pelo estado migratório dos indivíduos, pode-se supor que o Cinturão Metropolitano e Porto Alegre apresentem os maiores valores por terem maior proporção de migrante. Neste sentido, estariam representando os valores pressóricos deste último grupo. No entanto, quando as médias pressóricas dos naturais são comparadas às dos migrantes, encontra-se que elas são semelhantes (Tabela I).

Os migrantes intra-estrato diferem dos naturais pela experiência de terem migrado. Constituem-se num grupo que 
* $86 \%$ dos migrantes intra-estrato foram encontrados residindo no mesmo municipio de nascimento. permite isolar o efeito da migração, mantida a semelhança quanto ao local de nascimento e de residência.*

\section{GRÁFICO 1}

MÉDIAS DE PRESSĀO ARTERIAL DIASTÓLICA POR LOCAL DE RESIDÊNCIA E SEXO RIO GRANDE DO SUL, 1978

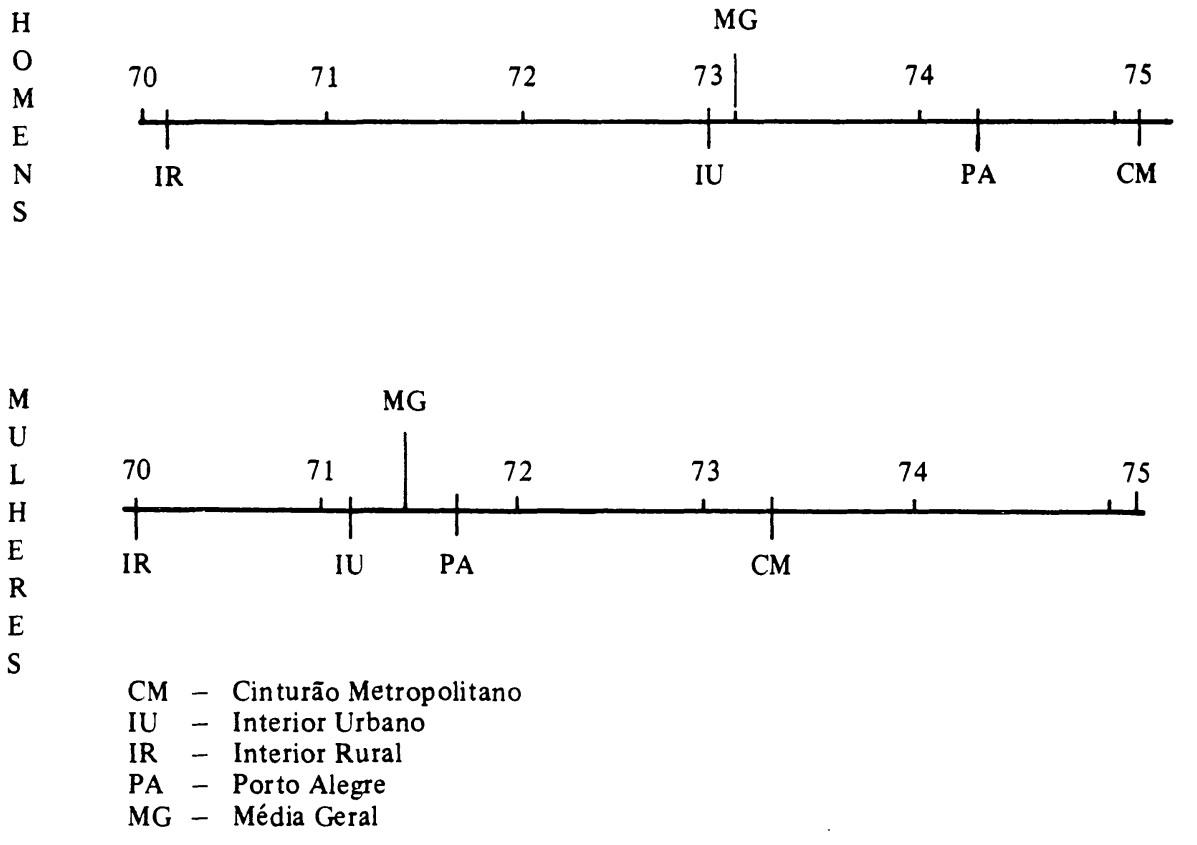

\section{TABELA I}

Contrastes das médias de pressão arterial diastólica, segundo o sexo, dos migrantes em relação aos naturais, controlados por idade e indice de Quetelet, Rio Grande do Sul, 1978.

\begin{tabular}{|c|c|c|c|c|c|}
\hline \multirow[b]{2}{*}{ Sexo } & \multicolumn{2}{|c|}{ Natural } & \multicolumn{2}{|c|}{ Migrante } & \multirow{2}{*}{$\frac{\text { Migrante-Natural }}{\beta^{* *}}$} \\
\hline & $\begin{array}{c}\text { No de } \\
\text { Indivíduos }\end{array}$ & $\begin{array}{l}\text { Médias } \\
\text { da PAD* }\end{array}$ & $\begin{array}{c}\mathrm{N} O \text { de } \\
\text { Indivíduos }\end{array}$ & $\begin{array}{l}\text { Médias } \\
\text { da PAD* }\end{array}$ & \\
\hline $\begin{array}{l}\text { Masculino } \\
\text { Feminino }\end{array}$ & $\begin{array}{l}615 \\
657\end{array}$ & $\begin{array}{l}71.3 \\
69.9\end{array}$ & $\begin{array}{l}1.302 \\
1.673\end{array}$ & $\begin{array}{l}73.7 \\
72.0\end{array}$ & $\begin{array}{r}-0.14 \\
0.16\end{array}$ \\
\hline
\end{tabular}

Diferenças não-significativas ao nível de $5 \%$.

* Médias brutas de pressão arterial diastólica.

** Contrastes das médias controladas por idade e índice de Quetelet. 
$\mathrm{Na}$ Tabela II, comparam-se as médias de pressão arterial diastólica dos naturais e migrantes intra-estrato. Observa-se que não existem diferenças entre os dois grupos em nenhum dos estratos. Ou seja, o fato de haver migrado não se refle. tiu em aumento da pressão arterial.

\section{TABELA II}

Contrastes das médias de pressão arterial diastólica, segundo o sexo, dos migrantes intra-estrato em relação aos naturais, controlados por idade e indice de Quetelet, Rio Grande so Sul, 1978.

\begin{tabular}{|c|c|c|c|c|c|c|c|}
\hline \multirow{2}{*}{$\begin{array}{c}\text { Local de } \\
\text { Nascimento }\end{array}$} & \multirow{2}{*}{$\begin{array}{c}\text { Local de } \\
\text { Residência }\end{array}$} & \multicolumn{2}{|c|}{ Masculino } & \multirow[b]{2}{*}{$\begin{array}{c}\text { Migrante } \\
- \text { Natural } \\
\beta^{* *}\end{array}$} & \multicolumn{2}{|c|}{ Feminino } & \multirow[b]{2}{*}{$\begin{array}{c}\text { Migrante } \\
\text {-Natural } \\
\beta^{* *}\end{array}$} \\
\hline & & $\begin{array}{l}\text { Médias } \\
\text { da PAD* }\end{array}$ & $\begin{array}{l}\text { Número de } \\
\text { Indivíduos }\end{array}$ & & $\begin{array}{c}\text { Médias } \\
\text { da PAD* }\end{array}$ & $\begin{array}{l}\text { Número de } \\
\text { Indivíduos }\end{array}$ & \\
\hline $\begin{array}{l}\text { Cinturão } \\
\text { Metropolitano }\end{array}$ & $\begin{array}{l}\text { Cinturão } \\
\text { Metropolitano }\end{array}$ & 72.2 & 38 & 0.78 & 74.6 & 32 & 3.70 \\
\hline $\begin{array}{l}\text { Interior } \\
\text { Urbano }\end{array}$ & $\begin{array}{l}\text { Interior } \\
\text { Urbano }\end{array}$ & 72.8 & 82 & 0.24 & 68.9 & 122 & 1.73 \\
\hline $\begin{array}{l}\text { Interior } \\
\text { Rural }\end{array}$ & $\begin{array}{l}\text { Interior } \\
\text { Rural }\end{array}$ & 70.2 & 249 & -0.73 & 70.8 & 277 & 1.10 \\
\hline Porto Alegre & Porto Alegre & 74.3 & 19 & -0.16 & 69.0 & 27 & -3.86 \\
\hline
\end{tabular}

Diferenças não-significativas ao nível de $5 \%$.

* Médias brutas da pressão arterial diastólica.

** Contrastes das médias controladas por idade e índice de Quetelet.

A Tabela III mostra os contrastes das médias de pressão arterial diastólica entre os naturais e migrantes intra-estratos. Como pode ser visto, não existem diferenças entre os dois grupos. Os migrantes, independente de onde se origi. nam, são semelhantes quanto à pressão arterial aos naturais do local para onde se dirigem.

Resta ainda verificar se os níveis pressóricos dos migrantes são diferentes dos naturais do local onde nasceram.

A Tabela IV mostra que os nascidos no Interior Rural que residem atualmente no Cinturão Metropolitano e Porto Alegre apresentam diferenças significativas $(P<0.05)$. Os migrantes que se deslocaram dentro dos estratos urbanos não se distanciaram dos níveis pressóricos dos naturais do local de origem, provavelmente porque as médias de pressão dos residentes nestes três estratos são semelhantes.

A Tabela V mostra as médias de pressão arterial diastólica dos migrantes oriundos da Área Rural que foram encontrados residindo em cidades, discriminados por tempo de residência.

Como pode ser visto, apenas os indivíduos que residem hă menos de um ano na área urbana têm níveis pressóricos mais baixos que os que lá moram. Este grupo tem também médias de pressão arterial diastólica semelhantes aos naturais da Área Rurạl. 
PESQUISA

\section{TABELA III}

Contrastes das médias de pressão arterial diastólica, segundo o sexo, dos migrantes, tomando como padräo os naturais em cada estrato, controlados por idade e indice de Quetelet, Rio Grande do Sul - 1978.

\begin{tabular}{|c|c|c|c|c|c|c|c|}
\hline \multirow{2}{*}{$\begin{array}{l}\text { Residência } \\
\text { Atual }\end{array}$} & \multirow{2}{*}{$\begin{array}{c}\text { Local de } \\
\text { Nascimento } \\
\text { dos Migrantes }\end{array}$} & \multicolumn{3}{|c|}{ Masculino } & \multicolumn{3}{|c|}{ Feminino } \\
\hline & & $\begin{array}{l}\text { Médias da } \\
\text { PAD* }\end{array}$ & $\begin{array}{l}\text { Número de } \\
\text { Indivíduos }\end{array}$ & $\begin{array}{c}\text { Migrante- } \\
\text { Natural } \\
\beta^{* *}\end{array}$ & $\begin{array}{l}\text { Médias da } \\
\text { PAD* }\end{array}$ & $\begin{array}{l}\text { Número de } \\
\text { Indivíduos }\end{array}$ & $\begin{array}{c}\text { Migrante- } \\
\text { Natural } \\
\beta^{* *}\end{array}$ \\
\hline $\begin{array}{l}\text { Cinturão } \\
\text { Metropolitano }\end{array}$ & $\begin{array}{l}\text { Interior Urbano } \\
\text { Interior Rural } \\
\text { Porto Alegre }\end{array}$ & $\begin{array}{l}77.1 \\
75.5 \\
77.1\end{array}$ & $\begin{array}{r}82 \\
261 \\
39\end{array}$ & $\begin{array}{l}2.74 \\
1.53 \\
3.56\end{array}$ & $\begin{array}{l}72.5 \\
73.5 \\
71.3\end{array}$ & $\begin{array}{r}102 \\
320 \\
47\end{array}$ & $\begin{array}{r}0.12 \\
0.16 \\
-1.18\end{array}$ \\
\hline $\begin{array}{l}\text { Interior } \\
\text { Urbano }\end{array}$ & Interior Rural & 73.3 & 241 & -1.62 & 72.5 & 345 & -0.16 \\
\hline $\begin{array}{l}\text { Interior } \\
\text { Rural }\end{array}$ & Interior Urbano & 67.3 & 11 & -3.82 & 67.3 & 12 & -2.38 \\
\hline Porto Alegre & $\begin{array}{l}\text { Cinturão } \\
\text { Metropolitano } \\
\text { Interior Urbano } \\
\text { Interior Rural }\end{array}$ & $\begin{array}{l}68.0 \\
74.1 \\
76.3\end{array}$ & $\begin{array}{r}11 \\
146 \\
123\end{array}$ & $\begin{array}{l}-6.10 \\
-1.18 \\
-0.18\end{array}$ & $\begin{array}{l}71.1 \\
71.2 \\
73.9\end{array}$ & $\begin{array}{r}18 \\
196 \\
175\end{array}$ & $\begin{array}{r}-2.75 \\
-0.88 \\
1.37\end{array}$ \\
\hline
\end{tabular}

Diferenças não-significativas ao nível de $5 \%$.

* Médias brutas de pressão arterial diastólica.

** Contrastes das médias controladas por idade e índice de Quetelet.

\section{TABELA IV}

Contraste das médias de pressāo arterial diastólica, segundo o sexo, dos migrantes, por local de origem, tomando como padrão os naturais do local de nascimento, controlados por idade e indice de Quetelet, Rio Grande do Sul, 1978

\begin{tabular}{|c|c|c|c|c|c|c|c|}
\hline \multirow{2}{*}{$\begin{array}{c}\text { Local de } \\
\text { Nascimento }\end{array}$} & \multirow{2}{*}{$\begin{array}{l}\text { Residência } \\
\text { atual dos } \\
\text { Migrantes }\end{array}$} & \multicolumn{3}{|c|}{ Masculino } & \multicolumn{3}{|c|}{ Feminino } \\
\hline & & $\begin{array}{l}\text { Médias } \\
\text { da PAD* }\end{array}$ & $\begin{array}{l}\text { Número de } \\
\text { Indivíduos }\end{array}$ & $\begin{array}{c}\text { Migrante- } \\
\text { Natural } \\
\beta^{* *}\end{array}$ & $\begin{array}{l}\text { Médias } \\
\text { da PAD* }\end{array}$ & $\begin{array}{l}\text { Número de } \\
\text { Indivíduos }\end{array}$ & $\begin{array}{c}\text { Migrante- } \\
\text { Natural } \\
\beta^{* *}\end{array}$ \\
\hline $\begin{array}{l}\text { Cinturão } \\
\text { Metropolitano }\end{array}$ & Porto Alegre & 68.0 & 11 & -4.96 & 71.1 & 18 & -3.70 \\
\hline Interior Urbano & $\begin{array}{l}\text { Cinturão } \\
\text { Metropolitano } \\
\text { Interior Rural } \\
\text { Porto Alegre }\end{array}$ & $\begin{array}{l}77.1 \\
67.3 \\
74.1\end{array}$ & $\begin{array}{r}82 \\
11 \\
146\end{array}$ & $\begin{array}{r}2.90 \\
-5.59 \\
0.12\end{array}$ & $\begin{array}{l}72.5 \\
67.3 \\
71.2\end{array}$ & $\begin{array}{r}102 \\
12 \\
196\end{array}$ & $\begin{array}{r}1.46 \\
-4.50 \\
-0.50\end{array}$ \\
\hline Interior Rural & $\begin{array}{l}\text { Cinturão } \\
\text { Metropolitano } \\
\text { Interior Urbano } \\
\text { Porto Alegre }\end{array}$ & $\begin{array}{l}75.5 \\
73.3 \\
76.3\end{array}$ & $\begin{array}{l}261 \\
241 \\
123\end{array}$ & $\begin{array}{l}3.62+ \\
0.15 \\
2.89+\end{array}$ & $\begin{array}{l}73.5 \\
72.5 \\
73.9\end{array}$ & $\begin{array}{l}320 \\
345 \\
175\end{array}$ & $\begin{array}{l}3.62+ \\
1.97 \\
3.88+\end{array}$ \\
\hline Porto Alegre & $\begin{array}{l}\text { Cinturão } \\
\text { Metropolitano }\end{array}$ & 77.1 & 39 & 2.42 & 71.3 & 47 & -0.23 \\
\hline
\end{tabular}

+ Diferenças significativas ao nível de $5 \%$.

* Médias brutas de pressão arterial diastólica.

** Contrastes das médias controladas por idade e indice de Quetelet. 


\section{TABELA V}

Contrastes das médias de pressão arterial diastolica dos migrantes procedentes da área rural e com residência atual em área urbana, tomando como padrão os residentes da área urbana, Rio Grande do Sul, 1978

\begin{tabular}{cccc}
\hline $\begin{array}{c}\text { Tempo } \\
\text { Residên cia em } \\
\text { Ärea Urbana }\end{array}$ & $\begin{array}{c}\text { Número de } \\
\text { Indivíduos }\end{array}$ & $\begin{array}{c}\text { Diferenças em } \\
\text { relação aos } \\
\text { Urbanos* }\end{array}$ & $\begin{array}{c}\text { Erro } \\
\text { Padrão }\end{array}$ \\
\hline Menos de 1 ano & 32 & $-6.03+$ & 2.21 \\
1 a 2 anos & 47 & -0.88 & 1.83 \\
3 a 5 anos & 87 & -0.57 & 1.36 \\
6 a 10 anos & 191 & -0.31 & 0.93 \\
11 a 20 anos & 236 & -0.10 & 0.76 \\
Mais de 20 anos & 406 & -0.52 & 0.68 \\
\hline
\end{tabular}

* Controlados por idade e índice de Quetelet

+Diferenças significativas ao nível de $1 \%$.

\section{DISCUSSÃO}

As migrações internas constituem-se em um movimento social da maior importância no Rio Grande do Sul. Os estratos de Porto Alegre e Cinturão Metropolitano são os principais pólos de concentração de migrantes. Dos migrantes que residem nestas duas áreas, a maioria nasceu na Ârea Rural; no entanto, cerca de $50 \%$ deles procederam de uma localidade urbana. Estes dados mostram que efetivamente a migração interna no Rio Grande do Sul se processou em etapas, sendo o último estágio os grandes centros urbanos, onde os migrantes chegam com alguma experiência acumulada em passagens anteriores por outras cidades menores ${ }^{13}$.

A Pressão A. Diastólica dos indivíduos que residem no Cinturão Metropolitano e Porto Alegre são semelhantes e mais elevadas que a dos residentes no Interior Urbano e Interior Rural, sendo o Interior Rural o que apresenta os menores valores. Os migrantes que são encontrados residindo em qualquer destes estratos apresentam os mesmos níveis pressóricos dos não-migrantes, independente do local de onde se originam. Assim, encontra-se, por exemplo, que os migrantes nascidos no Interior Rural, Interior Urbano e Porto Alegre que vão residir no Cinturão Metropolitano têm pressões arteriais semelhantes entre si e semelhantes aos não-migrantes do Cinturão Metropolitano. Em qualquer dos outros estratos, o mesmo fenômeno ocorre.

Os migrantes intra-estrato, como foi mostrado na Tabela II, também apresentam a Pressão A. Diastólica semelhante a dos não-migrantes do estrato correspondente. Estes da. dos indicam que a pressão arterial dos individuos que mo. ram no Rio Grande do Sul é muito mais influenciada pelas características do local de residência do que pela migração. 
Assim sendo, pode-se supor que a migração em si não foi capaz de reproduzir alterações na pressão sangüínea através de um estresse difuso de migrar. Os baixos valores de pressão arterial dos que migram dentro do estrato rural reforçam indubitavelmente o questionamento da migração como fator de risco para o aumento da pressão arterial no Rio Grande do Sul.

Por outro lado, o crescimento acelerado das cidades no Rio Grande do Sul, nos últimos quarenta anos, particularmente daquelas que compõem a Região Metropolitana de Porto Alegre, permite supor que os naturais são também, em sua grande maioria, filhos de migrantes nascidos no interior do Estado. Talvez isto contribua para uma maior identidade cultural dos migrantes com os padrões de vida desses novos ambientes.

Como já foi mostrado na Tabela IV, apenas os migrantes nascidos no Interior Rural que vão residir no Cinturão Metropolitano e Porto Alegre apresentam diferenças significativas na Pressão A. Diastólica, quando comparados com os seus pares não-migrantes do Interior Rural. Estes dados sugerem que a migração do Interior Rural para a Região Metropolitana é desfavorável, do ponto de vista da pressão arterial.

Neste estudo, encontram-se algumas evidências importantes que contrariam a idéia de seletividade da migração quanto à pressão arterial no Rio Grande do Sul: os migrantes nascidos no Interior Rural que são encontrados residindo em áreas urbanas apresentam níveis pressóricos crescentes, quando aumenta o tempo de permanência. $\mathrm{E}$, o que é mais interessante, os que residem há um ano ou menos nas cidades têm a mesma Pressão A. Diastólica dos residentes do Interior Rural. Os dados apresentados na Tabela V sugerem que o período crítico da adaptação da Pressão A. Diastólica dos migrantes rurais aos valores urbanos ocorre entre o segundo e o terceiro ano de permanência nas cidades. Um estudo realizado nas Regiões Metropolitanas do Brasil, inclusive a de Porto Alegre, mostra que as diferenças de nível de renda e tipos de ocupação entre migrantes e não-migrantes apenas são detectáveis nos dois primeiros anos, onde o migrante apresenta-se em situação de desvantagem. Em seguida, são superadas ${ }^{21}$.

Como já foi visto, no Rio Grande do Sul, a migração se processa em etapas, portanto espera-se que uma parcela de indivíduos que foram encontrados residindo no Interior Urbano se destine à Região Metropolitana. Sabe-se também que a Pressão A. Diastólica é mais baixa no Interior Rural, seguindo-se o Interior Urbano, Porto Alegre e Cinturão Metropolitano e que a Pressão Arterial dos migrantes não difere dos não-migrantes em nenhum dos estratos amostrais. 
Portanto, pode-se supor que vai se processando um aumento gradativo na Pressão Arterial dos migrantes que fazem o percurso migratório em etapas. Assim, as médias de Pressão A. Diastólica acompanham o processo adaptativo do migrante no seu deslocamento. Possivelmente o aparelho circulatório, através de relações de estresse continuadas, vai se deslocando em estado de prontidão, para conviver com os novos estímulos que se apresentam no novo ambiente.

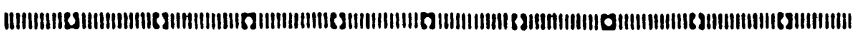

Este trabalho foi realizado no Estado do Rio Grande do Sul, Brasil, no ano de 1978.

Foram amostrados 2.056 domicilios, num total de 4.500 pessoas entre 20 e 74 anos de idade. A amostra foi composta por quatro estratos: Porto Alegre (capital), Cinturão Metropolitano (cidades em torno de Porto Alegre), Interior Urbano (sedes municipais) e Interior Rural.

Analisou-se a migração dentro e entre os quatro estratos amostrais e sua relação com a pressão arterial.

Os moradores da Área Rural apresentaram os mais baixos niveis pressóricos e os do Cinturão Metropolitano os mais altos.

As médias de pressão arterial diastólica dos naturais $e$ migrantes em cada estrato foram semelhantes.

Os migrantes nascidos na área nural que foram residir em Porto Alegre e Cinturão Metropolitano apresentaram médias pressóricas mais elevadas que os individuos naturais da área rural. Por outro lado, os individuos que migraram dentro do estrato rural (de uma área rural para outra) exibiram baixos valores de pressão arterial e não se mostraram diferentes dos naturais.

Importa ainda registrar que os migrantes oriundos da área rural que foram encontrados residindo em cidades apresentaram valores tensionais crescentes quando aumentava o tempo de permanência.

\section{REFERÊNCIAS BIBLIOGRÁFICAS}

1. CASSEL, J. Studies of hypertension in migrant. In: Epidemiology and control of hypertension. Miami, Symposia Specialists Medical Books, 1975. p. 41-62.

2. CHARVAT, J. et alii. Mental factors and cardiovascular disease. Cardiol., 44: 124-41, 1964. 
3. CHIANG, B.H. et alii. Owerweight and hypertension; a review. Circulation, $39: 403 \cdot 21,1969$.

4. COSTA, E.A. A cross-sectional survey of blood pressure in Rio Grande do Sul, Brasil. London, 1981. [Ph.D. - London School of Hygiene and Tropical Medicine].

5. CRUZ-COKE, R. et alii. Genetic ecology of hypertension. Clin. Sci. Molec. Med., 45 :55, 1973.

6. CRUZ-COKE, R. et alii. Influence of migration on blood pressure of easter islanders. Lancet, $1: 697-9$, 1964.

7. FREIS, E.D. Salt. Volume and the prevention of hypertension. Circulation, $53: 589.95,1976$.

8. FUNDAÇÃO DE ECONOMIA E ESTATÍSTICA. Migrações internas $R$. S. Indicadores Sociais, $R S$. Porto Alegre, 1976. número especial.

9. FUNDAÇÃO DE ECONOMIA E ESTATISTICA. 25 anos de economia gaúcha. Aspectos demográficos e suas relações com o desenvolvimento econômico do estado. Porto Alegre, 1977.

10. HENRY, J.P. \& CASSEL, J.C. Psychossocial factors in essential hypertension; recent epidemiologic and animal experimental evidence. Amer. J. Epidemiol., 90 (3) :171-200, 1969.

11. KANNEL, W.B. et alii. The relation of adiposity to blood pressure and development of hypertension; the Framingham Study. Ann. Inter. Med., 67 (2) $: 48.67,1967$.

12. KLEIN, C. H. Hipertensão arterial em estratos geo-econômicos no Rio Grande do Sul. Rio de Janeiro, 1984. [Tese de Mestrado, Escola Nacional de Saúde Pública, FIOCRUZ].

13. LEAL, M.C. Migrą̧ōes internas e pressão arterial no Rio Grande do Sul. Rio de Janeiro, 1981. [Tese de Mestrado, Escola Nacional de Saúde Pública, FIOCRUZ].

14. NADIM, A. et alii. Blood pressure and urban migration in Iran. Inter. J. Epidemiol., 7 (2) :131-80, 1978.

15. PRIOR, I.A.M. et alii. The Tokelau Island migrant study. Inter. J. Epidemiol., 3 (3) :225-32, 1974.

16. ROSE, G. Standardization of observers blood pressure measu rement. Lancet, l :673, 1975. 
17. SCOTCH, N.A. A preliminary report on the relation of socio-cultural factors to hypertension among the Zulu. Amer. N. Y. Acad. Sci., $84: 1.000-9,1960$.

18. SCOTCH, N.A. Sociocultural factors in the epidemiology of Zulu hypertension. Amer. J. Public Health, $53: 1.205-13,1963$.

19. SEYLE, H. The evolution of the stress concept; stress and cardiovascular disease. Amer. J. Cardiol., 26 :289-99, 1970.

20. SEYLE, H. Stress; a treatise based on the concepts of the general-adaptation-syndrome and the disease of adaptation. Canada, Acta Inc. Medical Publ., 1950.

21. SIMÕES, C.C. et alii. Contribuição ao estudo de migrações internas no Brasil; influência do nivel de desen. volvimento econômico sobre alguns diferenciais nas áreas metropolitanas de São Paulo, Rio de Janeiro, Porto Alegre e Recife. In: ENCONTRO BRASILEIRO DE ESTUDOS POPULACIONAIS; contribuições apresentadas. Rio de Janeiro, FIBGE, 1976. p. 211.22.

22. SIVE, P. H. et alii. Correlation of weight-height index with diastolic and with blood pressure. Brit. J. Prev. Soc. Med., $24: 201.4,1979$.

23. STRAMER, C.F. \& ERIZZLE, J.E. A computer program for analysis of data by general linear models. Int. of Statistics, 1960. mimeo [series n? 560].

24. WESSEN, A.F. The role of migrant studies in epidemiological research. Isr. J. Med. Sci., 7 (12) :1.584. 91, 1971. 